(C)2018 IEEE. Personal use of this material is permitted. Permission from IEEE must be obtained for all other uses, in any current or future media, including reprinting/republishing this material for advertising or promotional purposes, creating new collective works, for resale or redistribution to servers or lists, or reuse of any copyrighted component of this work in other works 


\section{Dual Kalman Filtering based GNSS Phase Tracking for Scintillation Mitigation}

\author{
Friederike Fohlmeister \\ Inst. for Communictations and Navigation \\ German Aerospace Center (DLR) \\ Wessling Germany \\ friederike.fohlmeister@dlr.de
}

\author{
Felix Antreich \\ Dep. of Teleinformatics Engineering \\ Federal University of Ceara (UFC) \\ Fortaleza, Brazil \\ antreich@ieee.org
}

\author{
Josef A. Nossek \\ Dep. of Teleinformatics Engineering \\ Federal University of Ceara (UFC) \\ Fortaleza, Brazil \\ Technische Universität München (TUM) \\ Munich, Germany \\ josef.a.nossek@tum.de
}

\begin{abstract}
This paper treats the problem of tracking the global navigation satellite system's (GNSS) signal phase under ionospheric scintillations and to generate an estimate of the scintillation phase and amplitude. In the case of ionospheric scintillations traditional phase locked loop (PLL) based GNSS receivers have problems to track the signal phase accurately. Moreover, these receivers do not estimate the scintillation phase and amplitude, which is evaluated in ionospheric monitoring applications. Therefore, Kalman filter based phase tracking algorithms have been proposed, which track line-of-sight (LOS) phase and scintillation amplitude and phase separately with different state space models. For modeling the scintillation phase and amplitude auto-regressive (AR) models can be used. In this case the parameters for the AR model have to be estimated together with the LOS and scintillation phase and amplitude. We propose to use a second Kalman filter for the estimation of the AR model parameters. This Kalman filter takes the scintillation phase and amplitude estimates from the first Kalman filter as measurement and the AR model parameters as system state. Simulation results for the global positioning system (GPS) show that the proposed algorithm achieves the same estimation performance for scintillation phase and amplitude as an algorithm with a priori knowledge on the AR model parameters.
\end{abstract}

\section{INTRODUCTION}

Global Navigation Satellite Systems (GNSS) are used in a wide variety of applications to provide a globally and permanently available position solution. However, in the case of ionospheric scintillations the signal of one or more satellites can be disturbed. Ionospheric scintillations are rapid fluctuations in the parameters of an electromagnetic wave, caused by ionospheric irregularities, i.e., an increase or depletion of the total electron content (TEC) of the ionosphere. These irregularities lead to a diffraction or refraction of the electro-magnetic wave traveling through the ionosphere, which leads to rapid fluctuations in the amplitude and phase of the received signal. On the one hand the decreased signal amplitude, increased phase noise and sudden phase drifts can degrade the accuracy of the pseudorange and carrier phase estimate, lead to cycle slips or induce loss of lock. This can directly degrade the position solution or make it unavailable if multiple satellites are affected. On the other hand the ionospheric effect on the signal can be used to trace back from the measured signal perturbations on the structure of the ionosphere. The GNSS receiver, in this case, acts as an ionospheric monitor which uses probes from different satellites at different points of the ionosphere. Ionospheric monitoring is not only essential from a scientific point of view but can be used to forecast and broadcast ionospheric threats, such that the user is aware of a possible degradation of the position solution.

The GNSS signal is commonly tracked by a closedloop phase locked-loop (PLL) and delay locked-loop (DLL). If the signal phase or amplitude shows deep fades due to ionospheric scintillations, the PLL may not be able to follow the signal phase and therefore go into a non-linear state or loose lock. In this case the tracked signal phase and amplitude is not purely representative of the line-of-sight (LOS) and scintillation effect but also includes non-linear receiver effects. This affects the receiver's positioning as well as ionospheric monitoring capabilities.

Different methods have been investigated to overcome this problem. To maintain lock under ionospheric scintillations with a classical PLL/ DLL tracking loop structure the PLL noise bandwidth can be adopted [1]. The noise bandwidth should be wide, to track rapidly changing carrier phases. In contrast, to tolerate amplitude scintillation and track signals with small amplitude the noise bandwidth should be chosen narrow. Receiver structures with adaptive loop noise bandwidth have been proposed [2], [3]. Also, a frequency locked loop (FLL) can be used to assist the PLL to maintain signal lock when the signal amplitude is small and a pure PLL structure would loose lock [1], [4]. Usually not all vissible satellites are affected by ionospheric scintillations. In this case vector tracking PLLs [5] can offer means to assist the tracking of signals affected by scintillations. A different approach is applied in so-called open loop or maximum likelihood (ML) structures. In this case, the phase error estimate is not fed-back into the tracking loop, i.e. the loop is not closed. Instead the signal is processed in batches. In each batch the phase is estimated with the ML principle [6], [7], i.e., searching for the signal parameters which maximize the probability density function for a given batch of received signal samples. Batch processing offers the advantage that the signal is not tracked based on its assumed dynamics and therefore also severe changes in phase and amplitude from one epoch to another can be tracked. A drawback of this 
approach is its increased computational complexity as the a priori information from the preceding batch is not fully used. If the receiver position is fixed, as it is the case for receiver monitoring stations, the ML approach can be assisted by the known receiver position [8] to reduce the computational effort.

To come up with a computationally more efficient tracking approach than ML methods, but with increased mathematical flexibility in comparison to the PLL, the Kalman filter can be used. In [9], [10] a three state Kalman filter with constant gain was proposed for phase tracking. In [11] an extended Kalman filter for joint tracking of code and carrier for weak signals, such as in the case of amplitude scintillations has been proposed. However, all of the mentioned approaches in general estimate the overall phase and amplitude. Such an estimate is based on the assumption that the signal amplitude and phase only follow the known LOS dynamics despite the scintillation amplitude and phase follow a process with a different characteristic. In the case of severe scintillations the overall amplitude and phase is therefore not tracked reliably. This can lead to cycle slips and loss of lock and the scintillation amplitude and phase cannot be fully extracted from the overall measurements. For ionospheric monitoring however, especially the scintillation amplitude and phase are of interest. Therefore, the overall phase and amplitude measurement is filtered to come up with a scintillation phase and amplitude estimate [12]. An alternative way to overcome this problem is to consider the scintillation phase and amplitude dynamics in the tracking loop.

Motivated by the low-pass characteristic of the power spectral density (PSD) of scintillation amplitude and phase [13]-[15] propose an autoregressive (AR) Kalman filtering approach. Scintillation phase and amplitude are incorporated into the Kalman filter as additional state-space variables which follow an AR model. Not only can the scintillation phase and amplitude, in this case, be directly used for monitoring of the ionosphere, also the LOS tracking becomes more robust. However, this concept requires not only a model of the signal's LOS dynamics, which are well known for static receivers but also the parameters of the AR model of the scintillation phase and amplitude. In [15] the AR parameters of the scintillation phase have to be estimated from the phase discriminator output with the Yule-Walker method.

The Yule-Walker method assumes that the scintillation amplitude and phase process is measured without noise. In the case of high LOS dynamics or additional distortion of the signal amplitude and phase, this cannot be ensured. In this paper, we therefore propose to use a second Kalman filter to estimate the process parameters of the AR model for scintillation amplitude and phase from the noisy scintillation amplitude and phase process. The scintillation phase and amplitude are estimated by the first Kalman filter. They are than used as measurements in the second Kalman filter to estimate the parameters of the respective AR process. This concept is referred to as dual Kalman filter [16]. The first Kalman filter is implemented as an extended Kalman filter which takes the correlation of the GNSS received signal with the local carrier signal replica as a measurement. This nonlinear measurement of the phase and frequency error is processed with an extended Kalman filter which uses the
AR model as a process model for the scintillation phase and amplitude. The second filter uses these scintillation phase and amplitude estimates as measurement inputs to estimate the AR model parameters. The LOS and scintillation phase error estimates are finally fed back into the carrier signal replica generator by a linear quadratic Gaussian control approach. This general control approach allows optimizing the tracking algorithm with respect to robustness and/or tracking accuracy.

The proposed algorithm is tested with computer simulations. Using the Cornell Scintillation Model (CSM) [17] we produce a time series which is representative for the statistics of amplitude and phase scintillation. The results show that the proposed dual Kalman filter approach can achieve the performance of a Kalman filter with a priori known AR model parameters under scintillation conditions.

\section{A. Notation}

In this paper, we define scalars, column vectors, and matrices with lowercase letters, lowercase bold letters, and uppercase bold letters, respectively. The transposition of a matrix $\boldsymbol{A}$ is denoted $\boldsymbol{A}^{\mathrm{T}}$. $\boldsymbol{I}_{A}$ denotes an identity matrix of dimension $A \times A$, and $\mathbf{0}_{A}$ denotes an all zero column vector of length $A$. $\operatorname{diag}(\boldsymbol{x}), \boldsymbol{x} \in \mathbb{R}^{A}$ produces a diagonal matrix of dimension $A \times A$ with the entries of $\boldsymbol{x}$ on its main diagonal, while blkdiag $\left(\boldsymbol{X}_{1}, \ldots, \boldsymbol{X}_{A}\right)$ denotes a block-diagonal matrix with matrices $\boldsymbol{X}_{1}, \ldots, \boldsymbol{X}_{A}$ stacked onto the main-diagonal.

\section{GNSS SIGNAL MODEL}

Consider the baseband received signal $y(t) \in \mathbb{C}$ with single sided bandwidth $B$ for one GNSS satellite

$$
y(t)=\gamma(t) e^{j(\phi(t)+\nu(t) t)} c(t-\tau(t))+\eta(t)
$$

with signal amplitude $\gamma(t) \in \mathbb{R}$, signal phase $\phi(t) \in \mathbb{R}$, angular Doppler frequency $\nu(t) \in \mathbb{R}$, signal time-delay $\tau(t) \in \mathbb{R}$ and periodically repeated, pseudo random (PR) code function $c(t) \in \mathbb{R}$ with $N_{\mathrm{c}}$ chips per code period. In (1) we include the Doppler effect as an offset on the carrier frequency, but neglect temporal dilatation effects. $\eta(t) \in \mathbb{C}$ is zero mean white Gaussian noise with variance $\sigma_{\eta}^{2}$. The GNSS phase tracking problem is to estimate the signal parameters $\gamma(t), \phi(t), \nu(t)$ from the measurements $y(t)$, while it is assumed that a code delay estimate $\hat{\tau}(t)$ is provided by an additional delay locked loop (DLL) and $\sigma_{\eta}^{2}$ is determined with a carrier-to-noise density ratio $\left(\mathrm{C} / \mathrm{N}_{0}\right)$ estimator [18]. In the case of ionospheric scintillations it is appropriate to split amplitude $\gamma(t)$ and phase $\phi(t)$ into $\operatorname{LOS} \gamma_{0}(t) \in \mathbb{R}, \phi_{0}(t) \in \mathbb{R}$ and scintillation contribution $\gamma_{\mathbf{S}}(t) \in \mathbb{R}, \phi_{\mathbf{S}}(t) \in \mathbb{R}$

$$
\begin{aligned}
& \gamma(t)=\gamma_{0}(t) \gamma_{\mathrm{S}}(t) \\
& \phi(t)=\phi_{0}(t)+\phi_{\mathrm{S}}(t) .
\end{aligned}
$$

As the coherence time of $\gamma_{0}(t)$ is large in comparison to the other parameters, $\gamma_{0}(t)$ is set to 1 without loss of generality and the effect of LOS amplitude attenuation for the estimation problem is reflected in the noise variance $\sigma_{\eta}^{2}$.

\section{A. Post-Correlation GNSS Signal Model}

After the signal $y(t)$ is sampled at a rate $f_{\mathrm{s}}=\frac{1}{T_{\mathrm{s}}}=2 B$ the discrete time signal model is

$$
y[n]=\gamma_{\mathrm{S}}[n] e^{j\left(\phi_{0}[n]+\phi_{\mathrm{S}}[n]+\nu[n] n T_{\mathrm{S}}\right)} c\left(n T_{\mathrm{S}}-\tau[n]\right)+\eta[n],
$$


where the short notation $x\left(n T_{\mathrm{s}}\right)=x[n]$ is used. Due to the typical distance between GNSS satellite and receiver, the received signal power and the signal-to-noise ratio (SNR) are low. Therefore, $N \in \mathbb{N}$ samples of the received signal are correlated with the samples of a local code replica. The parameters $\gamma_{\mathrm{S}}[n], \phi_{0}[n], \phi_{\mathrm{S}}[n], \nu[n] \tau[n]$, are assumed to be constant over the whole correlation time of length $T=N T_{\mathrm{s}}$ and $N$ is an integer multiple of $N_{\mathrm{c}}$. The measurement of the $k$-th correlation period reads

$$
\begin{aligned}
\tilde{z}[k] & =\frac{1}{N} \sum_{n=0}^{N-1} y[n] c\left(n T_{\mathrm{S}}-\hat{\tau}[k]\right) \\
& =\gamma_{\mathrm{S}}[k] \operatorname{sinc}\left(\frac{\nu[k] T}{2}\right) e^{j\left(\phi_{0}[k]+\phi_{\mathrm{S}}[k]+\frac{\nu[k]}{2} T\right)}+\tilde{v}[k],
\end{aligned}
$$

where the sinc function ${ }^{1}$ is

$$
\operatorname{sinc}(x)=\frac{\sin (x)}{x}
$$

and the noise variance of $\tilde{v}[k]$ is $\sigma^{2}=\frac{1}{N} \sigma_{\eta}^{2}$. The parameters to estimate are summarized in the vector

$$
\tilde{\boldsymbol{\xi}}[k]=\left[\phi_{0}[k], \nu[k], \phi_{\mathrm{s}}[k], \gamma_{\mathrm{S}}[k]\right]^{\mathrm{T}} \in \mathbb{R}^{4} .
$$

In the case of no a priori knowledge on $\tilde{\xi}[k]$ the ML estimator is the optimum estimator for estimating $\tilde{\xi}[k]$ from $\tilde{z}[k]$ [19]. However, the samples of $\gamma_{\mathrm{S}}[k], \phi_{0}[k], \phi_{\mathrm{S}}[k]$, and $\nu[k]$ do not evolve randomly from one epoch $k$ to the next epoch $k+1$, but are temporally correlated and can be represented with a state space model. Using this a priori knowledge on $\tilde{\xi}[k]$ a Bayesian estimator can be formulated.

\section{B. GNSS Signal Parameter State Space Models}

In the following we introduce the state space model for $\phi_{0}[k], \nu[k]$, and $\phi_{\mathrm{S}}[k]$, and $\gamma_{\mathrm{S}}[k]$. The LOS parameters can be described with a Wiener process while the scintillation parameters are described by an AR process.

1) LOS Phase State Space Model: The LOS signal phase $\phi_{0}[k]$ and Doppler frequency $\nu[k]$ are predominated by the LOS distance and velocity between satellite and receiver and the clock offset between satellite and receiver. Satellite-receiver distance [11] and clock offset [20] can be well modeled by a third order Wiener process. For simplicity we neglect the influence of the clock offset in the following. Signal phase $\phi_{0}[k]$ and Doppler frequency $\nu[k]$ are collected together with the angular Doppler drift $a[k] \in \mathbb{R}$ in the state vector

$$
\boldsymbol{x}_{0}[k]=\left[\phi_{0}[k], \nu[k], a[k]\right]^{\mathrm{T}} \in \mathbb{R}^{3} .
$$

This allows to formulate the discrete time state space model

$$
\begin{aligned}
\boldsymbol{x}_{0}[k+1] & =\boldsymbol{A}_{0} \boldsymbol{x}_{0}[k]+\boldsymbol{w}_{0}[k] \\
& =\left[\begin{array}{ccc}
1 & T & T^{2} / 2 \\
0 & 1 & T \\
0 & 0 & 1
\end{array}\right] \boldsymbol{x}_{0}[k]+\boldsymbol{w}_{0}[k] .
\end{aligned}
$$

\footnotetext{
${ }^{1}$ Exact derivations lead to the Dirichlet Kernel $\operatorname{di}(x)=\frac{\sin (x)}{\sin (x / N)}$ instead of $N \operatorname{sinc}(x)$. However, for large $N$ and $\nu[k]$ in the typical range between $-4 \cdot 2 \pi \mathrm{kHz}$ and $4 \cdot 2 \pi \mathrm{kHz}$ the Dirichlet Kernel is a scaled version of the unnormalized sinc function, i.e. $\operatorname{di}(x) \simeq N \operatorname{sinc}(x)=N \frac{\sin (x)}{x}$.
}

Note, that an exact measurement model (1) should also include the influence of $a[k]$. However, with respect to the measurement the influence of $a[k]$ is smaller than with respect to the state evolution. The process noise $\boldsymbol{w}_{0}[k] \in \mathbb{R}^{3}$ is zero mean Gaussian noise with covariance matrix

$$
\boldsymbol{Q}_{0}=\sigma_{\dot{a}}^{2}\left[\begin{array}{ccc}
\frac{T^{5}}{20} & \frac{T^{4}}{8} & \frac{T^{3}}{6} \\
\frac{T^{4}}{8} & \frac{T^{3}}{3} & \frac{T^{2}}{2} \\
\frac{T^{3}}{6} & \frac{T^{2}}{2} & T
\end{array}\right] \in \mathbb{R}^{3 \times 3},
$$

where $\sigma_{\dot{a}}^{2}$ is the variance of the angular Doppler drift rate in $\operatorname{rad}^{2} / \mathrm{s}^{5}$.

2) Scintillation State Space Models: Studies show that the scintillation phases $\phi_{\mathrm{S}}[k]$ follow a zero mean temporally correlated Gaussian process [21] with variance $\sigma_{\phi}^{2}$ while the scintillation amplitude $\gamma_{\mathrm{S}}[k]$ follows a temporally correlated Nakagami- $m$ distribution $^{2}$ [22]. The shape parameter $m \in \mathbb{R}$ is connected with the amplitude scintillation index by

$$
S_{4}=\sqrt{\frac{1}{m}}=\sqrt{\frac{\mathrm{E}\left[\gamma_{\mathrm{S}}[k]^{2}\right]-\mathrm{E}\left[\gamma_{\mathrm{S}}[k]\right]^{2}}{\mathrm{E}\left[\gamma_{\mathrm{S}}[k]\right]^{2}}} \in \mathbb{R} .
$$

The autocorrelation function of $\phi_{\mathrm{S}}[k]$ and $\gamma_{\mathrm{S}}[k]$ can be modeled with an $\operatorname{AR}(P)$ process of order $P[13]-[15]$, i.e.

$$
\begin{aligned}
\gamma_{\mathrm{S}}[k+1] & =\boldsymbol{a}_{\gamma}^{\mathrm{T}} \gamma_{\mathrm{S}}[k]+u_{\gamma}+w_{\gamma}[k] \\
\phi_{\mathrm{S}}[k+1] & =\boldsymbol{a}_{\phi}^{\mathrm{T}} \boldsymbol{\phi}_{\mathrm{S}}[k]+w_{\phi}[k],
\end{aligned}
$$

where $\boldsymbol{a}_{\gamma} \in \mathbb{R}^{P_{\gamma}}$ and $\boldsymbol{a}_{\phi} \in \mathbb{R}^{P_{\phi}}$ contain the AR process weights

$$
\begin{aligned}
& \boldsymbol{a}_{\gamma}=\left[a_{\gamma, 1} \ldots, a_{\gamma, P_{\gamma}}\right]^{\mathrm{T}} \\
& \boldsymbol{a}_{\phi}=\left[a_{\phi, 1} \ldots, a_{\phi, P_{\phi}}\right]^{\mathrm{T}},
\end{aligned}
$$

and $P_{\gamma} \in \mathbb{N}$ and $P_{\phi} \in \mathbb{N}$ are the orders of the AR process. The vectors

$$
\begin{aligned}
\gamma_{\mathrm{S}}[k] & =\left[\gamma_{\mathrm{S}}[k], \gamma_{\mathrm{S}}[k-1], \ldots, \gamma_{\mathrm{S}}\left[k-P_{\gamma}+1\right]\right]^{\mathrm{T}} \in \mathbb{R}^{P_{\gamma}} \\
\phi_{\mathrm{S}}[k] & =\left[\phi_{\mathrm{S}}[k], \phi_{\mathrm{S}}[k-1], \ldots, \phi_{\mathrm{S}}\left[k-P_{\phi}+1\right]\right]^{\mathrm{T}} \in \mathbb{R}^{P_{\phi}}
\end{aligned}
$$

contain $P_{\gamma}$ and $P_{\phi}$ samples of $\gamma_{\mathrm{S}}[k]$ and $\phi_{\mathrm{S}}[k]$, respectively of preceding epochs. $u_{\gamma} \in \mathbb{R}$ is a constant which accounts for the mean of $\gamma_{S}[k]$ and $w_{\gamma}[k] \in \mathbb{R}, w_{\phi}[k] \in \mathbb{R}$ are zero mean temporally white Gaussian noise with variance $\sigma_{\gamma}^{2}$ and $\sigma_{\phi}^{2}$. Using (14) and (15) the state space model for $\gamma_{\mathrm{S}}[k]$ and $\phi_{\mathrm{S}}[k]$ is

$$
\begin{aligned}
\gamma_{\mathrm{S}}[k+1] & =\boldsymbol{A}_{\gamma} \gamma_{\mathrm{S}}[k]+\boldsymbol{u}_{\gamma}+\boldsymbol{w}_{\gamma}[k] \\
\boldsymbol{\phi}_{\mathrm{S}}[k+1] & =\boldsymbol{A}_{\phi} \boldsymbol{\phi}_{\mathrm{S}}[k]+\boldsymbol{w}_{\phi}[k],
\end{aligned}
$$

where the AR process weights are included in the process matrices

$$
\boldsymbol{A}_{\boldsymbol{\gamma}}=\left[\begin{array}{c}
\boldsymbol{a}_{\gamma}^{\mathrm{T}} \\
{\left[\boldsymbol{I}_{P_{\gamma}-1}, \mathbf{0}_{P_{\gamma}-1}\right]}
\end{array}\right]
$$

and

$$
\boldsymbol{A}_{\boldsymbol{\phi}}=\left[\begin{array}{c}
\boldsymbol{a}_{\phi}^{\mathrm{T}} \\
{\left[\boldsymbol{I}_{P_{\phi}-1}, \boldsymbol{0}_{P_{\phi}-1}\right]}
\end{array}\right]
$$

\footnotetext{
${ }^{2}$ Probability density function of a Nakagami- $m$ distribution: $p(x)=$ $\frac{2 m^{m}}{\Gamma(m) \Omega^{m}} x^{2 m-1} \exp \left(-\frac{m}{\Omega} x^{2}\right), \forall x \geq 0, m \geq 0.5, \Omega>0$
} 
The noise terms $\boldsymbol{w}_{\boldsymbol{\gamma}}[k] \in \mathbb{R}^{P_{\gamma}}$ and $\boldsymbol{w}_{\phi}[k] \in \mathbb{R}^{P_{\phi}}$ are zero mean temporally white Gaussian noise with covariance matrices

$$
\boldsymbol{Q}_{\boldsymbol{\gamma}}=\operatorname{diag}\left(\left[\sigma_{\gamma}^{2}, \mathbf{0}_{P_{\boldsymbol{\gamma}}-1}^{\mathrm{T}}\right]^{\mathrm{T}}\right)
$$

and

$$
\boldsymbol{Q}_{\boldsymbol{\phi}}=\operatorname{diag}\left(\left[\sigma_{\phi}^{2}, \mathbf{0}_{P_{\phi}-1}^{\mathrm{T}}\right]^{\mathrm{T}}\right) .
$$

The state space model for $\gamma_{\mathrm{S}}[k]$ additionally contains the vector $\boldsymbol{u}_{\gamma}=\left[u_{\gamma}, \mathbf{0}_{P_{\gamma}-1}^{\mathrm{T}}\right]^{\mathrm{T}} \in \mathbb{R}^{P_{\gamma}}$.

3) Combined GNSS Signal Parameter State Space Model: Combining (10), (20), and (21) yields the state space vector $\overline{\boldsymbol{\xi}}[k] \in \mathbb{R}^{3+P_{\phi}+P_{\gamma}}$, which contains all parameters of $\tilde{\boldsymbol{\xi}}[k]$ and additionally contains parameters to express the propagation process of the parameters and follows the state space model

$$
\overline{\boldsymbol{\xi}}[k+1]=\boldsymbol{A} \overline{\boldsymbol{\xi}}[k]+\boldsymbol{u}_{\bar{\xi}}[k]+\boldsymbol{w}[k] .
$$

The state vector $\overline{\boldsymbol{\xi}}[k]$ and control vector $\boldsymbol{u}_{\overline{\boldsymbol{\xi}}}[k]$ are

$$
\begin{aligned}
\overline{\boldsymbol{\xi}}[k] & =\left[\boldsymbol{x}_{0}[k]^{\mathrm{T}}, \boldsymbol{\phi}_{\mathrm{S}}[k]^{\mathrm{T}}, \boldsymbol{\gamma}_{\mathrm{S}}[k]^{\mathrm{T}}\right]^{\mathrm{T}} \\
\boldsymbol{u}_{\bar{\xi}}[k] & =\left[\mathbf{0}_{3}^{\mathrm{T}}, \mathbf{0}_{P_{\boldsymbol{\phi}}}^{\mathrm{T}}, \boldsymbol{u}_{\gamma}[k]^{\mathrm{T}}\right]^{\mathrm{T}}
\end{aligned}
$$

and the process matrix $\boldsymbol{A}$ can be expressed by

$$
\boldsymbol{A}=\operatorname{blkdiag}\left(\boldsymbol{A}_{0}, \boldsymbol{A}_{\boldsymbol{\gamma}}, \boldsymbol{A}_{\boldsymbol{\phi}}\right) .
$$

The noise covariance matrix of $\boldsymbol{w}[k]$ is

$$
\boldsymbol{Q}=\operatorname{blkdiag}\left(\boldsymbol{Q}_{0}, \boldsymbol{Q}_{\boldsymbol{\gamma}}, \boldsymbol{Q}_{\boldsymbol{\phi}}\right) \text {. }
$$

\section{GNSS RECEIVER MODEL}

Given a linear state space model with Gaussian noise, such as (26) and a linear measurement equation with Gaussian noise for the state space vector, the Kalman filter is the optimum estimator for the parameter vector in the maximum a posteriori sense. However, as (6) is nonlinear in $\boldsymbol{\xi}[k]$ the measurement equation has to be linearized, i.e., the extended Kalman filter has to be used. The extended Kalman filter linearizes the nonlinear model around the reference trajectory and than operates the Kalman filter on the linear model. In contrast to the Kalman filter the extended Kalman filter is in general not optimal. As the linearization depends on the system state and must be calculated at run-time, the computational effort is larger than in the linear case. Additionally, the derivatives with respect to $\phi_{0}[k], \phi_{\mathrm{S}}[k]$ and $\nu[k]$ are nonlinear and include a $2 \pi$ ambiguity. To overcome these problems the received signal $y[n]$ is not only correlated with a code replica, but also with a phase replica such that the tracking problem is formulated in the error space of the parameters $\phi_{0}[k], \nu[k], a[k]$ and $\phi_{\mathrm{S}}[k]$, i.e., the errors

$$
\begin{aligned}
e_{\phi_{0}}[k] & =\phi_{0}[k]-\hat{\phi}_{0}[k] \quad \in \mathbb{R} \\
e_{\nu}[k] & =\nu[k]-\hat{\nu}[k] \quad \in \mathbb{R} \\
e_{a}[k] & =a[k]-\hat{a}[k] \quad \in \mathbb{R} \\
e_{\phi_{\mathrm{S}}}[k] & =\phi_{\mathrm{S}}[k]-\hat{\phi}_{\mathrm{S}}[k] \quad \in \mathbb{R}
\end{aligned}
$$

are tracked where $\hat{\phi}_{0}[k] \in \mathbb{R}, \hat{\nu}[k] \in \mathbb{R}, \hat{a}[k] \in \mathbb{R}, \hat{\phi}_{\mathrm{S}}[k] \in \mathbb{R}$ are the parameters of a phase replica and chosen such that the errors are close to zero.
1) GNSS Receiver Measurement Model: After correlation with the code and carrier replica signal the measurements read

$$
\begin{aligned}
z[k] & =\frac{1}{N} \sum_{n=0}^{N-1} y[n] c(n T-\hat{\tau}[k]) e^{-j\left(\hat{\phi}_{0}[k]+\hat{\phi}_{S}[k]+n T \hat{\nu}[k]\right)} \\
& =\gamma_{\mathrm{S}}[k] \operatorname{sinc}\left(\frac{e_{\nu}[k] T}{2}\right) e^{j\left(e_{\phi_{0}}[k]+e_{\phi_{\mathrm{S}}}[k]+\frac{e_{\nu}[k] T}{2}\right)}+v[k]
\end{aligned}
$$

and the problem is given by estimating the state vector

$$
\begin{aligned}
\boldsymbol{\xi}[k] & =\left[e_{\phi_{0}}[k], e_{\nu}[k], e_{a}[k], \boldsymbol{e}_{\phi_{\mathrm{S}}}^{\mathrm{T}}[k], \gamma_{\mathrm{S}}^{\mathrm{T}}[k]\right]^{\mathrm{T}} \\
& =\left[\boldsymbol{e}^{\mathrm{T}}[k], \gamma_{\mathrm{S}}^{\mathrm{T}}[k]\right]^{\mathrm{T}} \in \mathbb{R}^{3+P_{\phi}+P_{\gamma}}
\end{aligned}
$$

from the measurements $z[k]$, where

$$
\boldsymbol{e}_{\phi_{\mathrm{S}}}[k]=\left[e_{\phi_{\mathrm{S}}}[k], \ldots, e_{\phi_{\mathrm{S}}}\left[k-P_{\phi}+1\right]\right]^{\mathrm{T}} \in \mathbb{R}^{P_{\phi}} .
$$

To account for the real valued parameters, the measurements are expressed in the real valued augmented representation

$$
\begin{aligned}
& \boldsymbol{z}[k]=[\operatorname{Re}(z[k]), \operatorname{Im}(z[k])]^{\mathrm{T}} \in \mathbb{R}^{2} \\
& =\boldsymbol{h}(\boldsymbol{\xi}[k])+\boldsymbol{v}[k],
\end{aligned}
$$

where $\boldsymbol{v}[k]$ is Gaussian noise with covariance matrix

$$
\boldsymbol{R}=0.5 \sigma^{2} \boldsymbol{I}_{2}
$$

2) Replica Signal State Space Model: The replica parameters $\hat{\phi}_{0}[k], \hat{\nu}[k], \hat{a}[k], \hat{\phi}_{\mathrm{S}}[k]$ follow a similiar state space model as their true counterparts $\phi_{0}[k], \nu[k], a[k]$ and $\phi_{\mathrm{S}}[k]$, and are comprised in the state space vector $\boldsymbol{x}_{\mathrm{NCO}}[k] \in \mathbb{R}^{3+P_{\phi}}$. The subscript $N C O$ accounts for the implementation of the carrier replica in a receiver with a numerically controlled oscillator (NCO). The replica parameters state space model is

$$
\boldsymbol{x}_{\mathrm{NCO}}[k+1]=\boldsymbol{A}_{\mathrm{NCO}} \boldsymbol{x}_{\mathrm{NCO}}[k]+\boldsymbol{u}_{\mathrm{NCO}}[k],
$$

where

$$
\begin{aligned}
\boldsymbol{x}_{\mathrm{NCO}}[k] & =\left[\hat{\phi}_{0}[k], \hat{\nu}[k], \hat{a}[k], \hat{\boldsymbol{\phi}}_{\mathrm{s}}[k]^{\mathrm{T}}\right]^{\mathrm{T}} \\
\boldsymbol{A}_{\mathrm{NCO}} & =\operatorname{blkdiag}\left(\boldsymbol{A}_{0}, \boldsymbol{A}_{\phi}\right) \\
\boldsymbol{u}_{\mathrm{NCO}}[k] & =\left[u_{\phi_{0}}[k], u_{\nu}[k], u_{a}[k], \boldsymbol{u}_{\phi_{\mathrm{s}}}[k]^{\mathrm{T}}\right]^{\mathrm{T}},
\end{aligned}
$$

and

$$
\hat{\phi}_{\mathrm{s}}[k]=\left[\hat{\phi}_{\mathrm{s}}[k], \ldots, \hat{\phi}_{\mathrm{s}}\left[k-P_{\phi}+1\right]\right]^{\mathrm{T}} \in \mathbb{R}^{P_{\phi}} .
$$

The replica parameters are driven by a deterministic control vector $\boldsymbol{u}_{\mathrm{NCO}}[k]$ instead of random noise. The control vector describes the update from time instance $k$ to $k+1$. $\boldsymbol{u}_{\mathrm{NCO}}[k]$ must be chosen such that $e_{\phi_{0}}[k], e_{\phi_{\mathrm{S}}}[k], e_{\nu}[k]$ are small.

3) GNSS Receiver State Space Model: The state space model for $\boldsymbol{\xi}[k]$ is given by

$$
\boldsymbol{\xi}[k+1]=\boldsymbol{A} \boldsymbol{\xi}[k]+\boldsymbol{B} \boldsymbol{u}[k]+\boldsymbol{w}[k],
$$

where $\boldsymbol{A}$ is given in (29), the covariance of $\boldsymbol{w}[k]$ is given in (30), the control vector is

$$
\begin{aligned}
\boldsymbol{u}[k] & =\left[u_{\phi_{0}}[k], u_{\nu}[k], u_{a}[k], \boldsymbol{u}_{\phi_{\mathrm{s}}}[k]^{\mathrm{T}}, \boldsymbol{u}_{\gamma}^{\mathrm{T}}\right]^{\mathrm{T}} \\
& =\left[\boldsymbol{u}_{\mathrm{NCO}}[k]^{\mathrm{T}}, \boldsymbol{u}_{\gamma}^{\mathrm{T}}\right]^{\mathrm{T}} .
\end{aligned}
$$

and the coupling matrix $\boldsymbol{B}$ is

$$
\boldsymbol{B}=\operatorname{blkdiag}\left(-\boldsymbol{I}_{3+P_{\phi}}, \boldsymbol{I}_{P_{\gamma}}\right) .
$$




\section{GNSS Signal Parameter Tracking Problem}

Using the measurement model (41) and the process model (48) the GNSS phase tracking problem has two goals:

1) Estimate $\gamma_{S}[k]$ in the optimum a posteriori sense

2) Determine $\boldsymbol{u}_{\mathrm{NCO}}[k]$, such that the error between replica states and true states $\boldsymbol{e}[k]$ is minimized.

The problem $\mathcal{P}$ is described on the top of the next page in (52)-(55). The first part of $\mathcal{P},(52)$, formulates the a posteriori estimation problem for $\gamma_{\mathrm{S}}[k]$ with $\boldsymbol{P}_{\gamma}[0]$ being the initial error covariance of $\gamma_{\mathrm{S}}[0]$. As $\boldsymbol{h}(\boldsymbol{\xi}[k])$ is linear in $\gamma_{\mathrm{S}}[k]$ the problem is solved by the Kalman filter in the optimal sense if all other parameters of $\boldsymbol{\xi}[k]$ are perfectly known. The second part of $\mathcal{P},(53)$, aims to find a feasible control strategy $\boldsymbol{u}_{\mathrm{NCO}}[1], \ldots, \boldsymbol{u}_{\mathrm{NCO}}[K]$, which minimizes the expected value of the weighted quadratic error vector $\boldsymbol{e}[k]$ as well as the control effort. The weighting matrix $\boldsymbol{S}_{1} \in \mathbb{R}^{\left(3+P_{\boldsymbol{\phi}}\right) \times\left(3+P_{\boldsymbol{\phi}}\right)}$ is symmetric and positive semi-definite and the weighting matrix $\boldsymbol{S}_{2} \in \mathbb{R}^{\left(3+P_{\boldsymbol{\phi}}\right) \times\left(3+P_{\boldsymbol{\phi}}\right)}$ is symmetric and positive definite. The matrices are chosen such that they reflect an upper bound on the error $\boldsymbol{e}[k]$ and the control effort of $\boldsymbol{u}_{\mathrm{NCO}}[k]$ [23]. If $\boldsymbol{h}(\boldsymbol{\xi}[k])$ was linear in $\boldsymbol{e}[k]$, the design of the controller $\boldsymbol{u}_{\mathrm{NCO}}[k]$ and the estimation of $e[k]$ can be separated, [23], i.e., the optimum $\boldsymbol{u}_{\mathrm{NCO}}[k]$ are given by a linear quadratic regulator (LQR), while the estimates of $e[k]$ are found with a Kalman filter. In [24] it has been shown that for nonlinear measurement equations, the separation principle is still applicable. However, the Kalman filter is in this case not applicable to design the estimator. Alternatively, the extended Kalman filter is applied. Using the approximation $\boldsymbol{e}[k] \approx \mathbf{0}_{3+P_{\phi}}$ the linearization can be calculated in advance and $\boldsymbol{e}[k]$ and $\gamma_{\mathrm{S}}[k]$, i.e., $\boldsymbol{\xi}[k]$, can be estimated with the same Kalman filter. In the following we shortly describe the LQR for the determination of $\boldsymbol{u}_{\mathrm{NCO}}[k]$ and the Kalman filter for the estimation of $\boldsymbol{\xi}[k]$.

1) LQR Design for Signal Parameter Tracking: The optimum $\boldsymbol{u}_{\mathrm{NCO}}[k]$ is given by the LQR

$$
\boldsymbol{u}_{\mathrm{NCO}}[k]=-\boldsymbol{L} \boldsymbol{e}[k],
$$

where $\boldsymbol{L} \in \mathbb{R}^{\left(3+P_{\boldsymbol{\phi}}\right) \times\left(3+P_{\boldsymbol{\phi}}\right)}$ is the feedback gain matrix

$$
\boldsymbol{L}=-\left(\boldsymbol{T}+\boldsymbol{S}_{2}\right)^{-1} \boldsymbol{T} \boldsymbol{A}_{\mathrm{NCO}},
$$

and $\boldsymbol{T} \in \mathbb{R}^{\left(3+P_{\boldsymbol{\phi}}\right) \times\left(3+P_{\boldsymbol{\phi}}\right)}$ is determined by the discrete time algebraic Riccati equation [23]

$$
\boldsymbol{T}=\boldsymbol{A}_{\mathrm{NCO}}^{\mathrm{T}}\left(\boldsymbol{T}-\boldsymbol{T}\left(\boldsymbol{T}+\boldsymbol{S}_{2}\right)^{-1} \boldsymbol{T}\right) \boldsymbol{A}_{\mathrm{NCO}}+\boldsymbol{S}_{1} .
$$

A solution to (58) can be found by iterating on the equation.

\section{A. Extended Kalman Filter for Signal Parameter Tracking}

The estimation problem for both parameters $\gamma_{\mathrm{S}}[k]$ and $\boldsymbol{e}[k]$, i.e., $\boldsymbol{\xi}[k]$ can be solved with the extended Kalman filter. The extended Kalman filter yields the estimates

$$
\hat{\boldsymbol{\xi}}[k]=\hat{\boldsymbol{\xi}}[k \mid k-1]+\boldsymbol{K}[k] \boldsymbol{r}[k],
$$

where $\hat{\boldsymbol{\xi}}[k \mid k-1]$ is the a priori estimate of $\hat{\boldsymbol{\xi}}[k]$

$$
\hat{\boldsymbol{\xi}}[k \mid k-1]=\boldsymbol{A} \hat{\boldsymbol{\xi}}[k-1]+\boldsymbol{u}[k-1],
$$

$\boldsymbol{r}[k]$ is the measurement residual

$$
\boldsymbol{r}[k]=\boldsymbol{z}[k]-\boldsymbol{h}(\hat{\boldsymbol{x}}[k \mid k-1]),
$$

and $\boldsymbol{K}[k]$ is the Kalman gain matrix

$\boldsymbol{K}[k]=\boldsymbol{P}[k \mid k-1] \boldsymbol{H}^{\mathrm{T}}[k]\left(\boldsymbol{H}[k] \boldsymbol{P}[k \mid k-1] \boldsymbol{H}^{\mathrm{T}}[k]+\boldsymbol{R}\right)^{-1}$,

where $\boldsymbol{R}$ is given in (42) and $\boldsymbol{H}[k]$ is the Jacobian matrix of $\boldsymbol{h}(\boldsymbol{\xi}[k])$ evaluated at $\hat{\boldsymbol{\xi}}[k \mid k-1]$. For the calculation of $\boldsymbol{h}(\boldsymbol{\xi}[k])$ and $\boldsymbol{H}[k]$ we use $\boldsymbol{e}[k]=\mathbf{0}_{3+P_{\phi}}$, which reduces the computational effort. The a priori error covariance estimate $\boldsymbol{P}[k \mid k-1]$ is defined

$$
\boldsymbol{P}[k \mid k-1]=\boldsymbol{A} \boldsymbol{P}[k-1] \boldsymbol{A}^{\mathrm{T}}+\boldsymbol{Q},
$$

where the error covariance matrix estimate $\boldsymbol{P}[0]$ is the covariance of $\boldsymbol{\xi}[0]$ and the following $\boldsymbol{P}[k]$ are given by

$$
\boldsymbol{P}[k]=(\boldsymbol{I}-\boldsymbol{K}[k] \boldsymbol{H}[k]) \boldsymbol{P}[k \mid k-1] .
$$

\section{AR PARAMETER Estimation PROBlem}

The AR parameter vectors $\boldsymbol{a}_{\phi}$ and $\boldsymbol{a}_{\boldsymbol{\gamma}}$, the deterministic input $u_{\gamma}$, and the driving noise variances $\sigma_{\gamma}^{2}, \sigma_{\phi}^{2}$ are in general unknown. As the scintillation intensity changes with the TEC of the ionosphere, the parameters are also time-variant, even though their coherence time is larger than $N T$ and therefore the time invariant notation in the sections before is justified. When discussing the estimation of the AR parameters we nevertheless have to use the time-variant notation and the they have to be estimated together with the state estimates $\hat{\boldsymbol{\xi}}[k]$ at run-time. In this paper we propose to use a second Kalman filter to estimate the AR process parameters. The approach is depicted in Fig. 1. The received signal $y[k, n]$ is correlated with the code replica $c(n T-\hat{\tau}[k])$ and the carrier replica $e^{j\left(\hat{\phi}_{0}[k]+\hat{\phi}_{S}[k]+\hat{\nu}[k] n T\right)}$. The resulting signal $\boldsymbol{z}[k]$ is the measurement for the first Kalman filter Process $K F$. This Kalman filter estimates the state estimates $\boldsymbol{\xi}[k]$ as given in (59)-(64). The scintillation phase and amplitude estimates $\hat{\phi}_{\mathrm{S}}$ and $\hat{\gamma}_{\mathrm{S}}[k]$ are the measurements for the second Kalman filter Parameter $K F$. This Kalman filter estimates the scintillation parameters $\boldsymbol{a}_{\gamma}, \boldsymbol{a}_{\phi}, u_{\gamma}, \sigma_{\gamma}^{2}$, and $\sigma_{\phi}^{2}$. The scintillation parameter estimates are then fed into the LQR solver, the first Kalman filter and the carrier replica generator $N C O$. The Kalman filter estimates the AR parameters for $\hat{\phi}_{\mathrm{S}}[k]$ and $\hat{\gamma}_{\mathrm{S}}[k]$ separately, but with the same technique. To keep the derivation short, the Kalman filter is derived in general for both parameters $\hat{\phi}_{\mathrm{S}}[k]$ and $\hat{\gamma}_{\mathrm{S}}[k]$, and the index $\mathrm{S}$ accounts for either of the two parameters.

\section{A. AR Parameter Process Model}

The process model for the AR parameter vectors $\boldsymbol{x}_{\mathrm{S}}[k]$ is

$$
\boldsymbol{x}_{\mathrm{S}}[k]=\boldsymbol{x}_{\mathrm{S}}[k-1]+\boldsymbol{w}_{\mathrm{S}}[k],
$$

where the parameter vector is defined

$$
\boldsymbol{x}_{\mathrm{S}}[k]= \begin{cases}\phi_{\mathrm{S}}[k]: & \boldsymbol{a}_{\boldsymbol{\phi}}[k] \\ \gamma_{\mathrm{S}}[k]: & {\left[\boldsymbol{a}_{\boldsymbol{\gamma}}[k]^{\mathrm{T}}, u_{\gamma}[k]\right]^{\mathrm{T}} .}\end{cases}
$$

The noise term $\boldsymbol{w}_{\mathrm{S}}[k]$ is Gaussian with covariance matrices $\boldsymbol{Q}_{\gamma, \mathrm{AR}}$ or $\boldsymbol{Q}_{\phi, \mathrm{AR}}$, respectively, to account for the time variance of the scintillation process ${ }^{3}$.

\footnotetext{
${ }^{3} \boldsymbol{Q}_{\gamma, \mathrm{AR}}$, and $\boldsymbol{Q}_{\phi, \mathrm{AR}}$ can be used as tuning parameters. In the work at hand the parameter process covariance matrices are chosen as scaled identity matrices $\boldsymbol{Q}_{\gamma, \mathrm{AR}}=\mu_{\gamma} \boldsymbol{I}_{P_{\gamma}}$ and $\boldsymbol{Q}_{\phi, \mathrm{AR}}=\mu_{\phi} \boldsymbol{I}_{P_{\phi}}$, where $\mu_{\gamma} \ll 1$ and $\mu_{\phi} \ll 1$
} 


$$
\begin{aligned}
& \mathcal{P}: \quad \min _{\gamma_{S}[1], \ldots, \gamma_{S}[K]} \boldsymbol{\gamma}_{\mathrm{S}}^{\mathrm{T}}[0] \boldsymbol{P}_{\gamma}^{-1}[0] \boldsymbol{\gamma}_{\mathrm{S}}[0]+\sum_{k=0}^{K-1} \boldsymbol{w}_{\gamma}^{\mathrm{T}}[k] \boldsymbol{Q}_{\gamma}^{-1} \boldsymbol{w}_{\gamma}[k]+\sum_{k=1}^{K} \boldsymbol{v}^{\mathrm{T}}[k] \boldsymbol{R}^{-1} \boldsymbol{v}[k]
\end{aligned}
$$

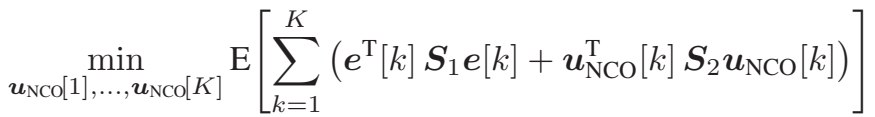

$$
\begin{aligned}
& \text { s.t. } \boldsymbol{\xi}[k]=\boldsymbol{A} \boldsymbol{\xi}[k-1]+\boldsymbol{B} \boldsymbol{u}[k]+\boldsymbol{w}[k] \\
& \boldsymbol{z}[k]=\boldsymbol{h}(\boldsymbol{\xi}[k])+\boldsymbol{v}[k],
\end{aligned}
$$

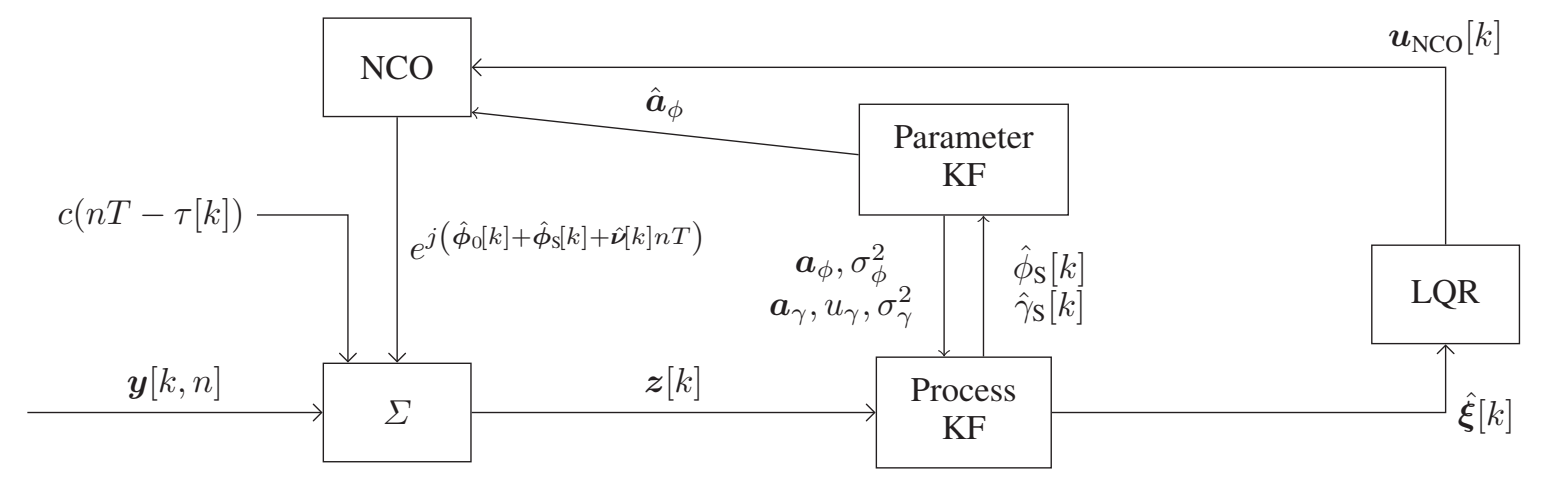

Fig. 1. Dual Kalman Filter Approach

\section{B. AR Parameter Measurement Model}

The estimates of $\gamma_{\mathrm{S}}[k]$ and $\phi_{\mathrm{S}}[k]$ provided by the primal Kalman filter are used as measurements in the second Kalman filter. Using (59) and (60) we can define the following measurement equation for the AR process parameters

$$
\begin{aligned}
z_{\mathrm{S}}[k] & =\boldsymbol{\Pi}_{S}^{\mathrm{T}}(\boldsymbol{A}[k] \hat{\boldsymbol{\xi}}[k-1]+\boldsymbol{u}[k]+\boldsymbol{K}[k] \boldsymbol{r}[k]) \\
& =\boldsymbol{h}_{\mathrm{S}}[k] \boldsymbol{x}_{\mathrm{S}}[k]+v_{\mathrm{S}}[k]
\end{aligned}
$$

where the measurement $z_{\mathrm{S}}[k] \in \mathbb{R}$ accounts for $\gamma_{\mathrm{S}}[k]$ and $\phi_{\mathrm{S}}[k]$, respectively. The rows which account for these measurements are selected from the state space vector of the primal Kalman filter $\boldsymbol{\xi}[k]$ with the selection matrices

$$
\boldsymbol{\Pi}_{S}=\left\{\begin{array}{ll}
\phi_{\mathrm{S}}[k]: & {\left[\mathbf{0}_{3}^{\mathrm{T}}, 1, \mathbf{0}_{P_{\phi}-1+P_{\gamma}}^{\mathrm{T}}\right]^{\mathrm{T}} \in \mathbb{R}^{3+P_{\gamma}+P_{\phi}}} \\
\gamma_{\mathrm{S}}[k]: & {\left[\mathbf{0}_{3+P_{\phi}}^{\mathrm{T}}, 1, \mathbf{0}_{P_{\gamma}-1}^{\mathrm{T}}\right]^{\mathrm{T}} \in \mathbb{R}^{3+P_{\gamma}+P_{\phi}}}
\end{array} .\right.
$$

The measurement matrices are defined as

$$
\boldsymbol{h}_{\mathrm{S}}[k]= \begin{cases}\phi_{\mathrm{S}}[k]: & \hat{\boldsymbol{\phi}}[k-1] \in \mathbb{R}^{P_{\phi}} \\ \gamma_{\mathrm{S}}[k]: & {\left[\hat{\gamma}^{\mathrm{T}}[k-1], 1\right]^{\mathrm{T}} \in \mathbb{R}^{\left(P_{\gamma}+1\right)}}\end{cases}
$$

and $v_{\mathrm{S}}[k]$ is temporally white Gaussian noise with variance

$$
\sigma_{\mathrm{S}}^{2}=\boldsymbol{\Pi}_{\mathrm{S}}^{\mathrm{T}} \boldsymbol{K}[k] \boldsymbol{S}[k] \boldsymbol{K}^{\mathrm{T}}[k] \boldsymbol{\Pi}_{\mathrm{S}},
$$

where $S[k]$ is the covariance matrix of the measurement residual of the primal Kalman filter $\boldsymbol{r}[k]$, i.e.,

$$
\boldsymbol{S}[k]=\boldsymbol{H}[k] \boldsymbol{P}[k \mid k-1] \boldsymbol{H}^{\mathrm{T}}[k]+\boldsymbol{R} .
$$

\section{AR Parameter Kalman Filter Estimation}

Using the measurement equations (68) and the process equation (65) the Kalman filter estimates for $x_{\mathrm{S}}[k]$ can be formulated following the general Kalman filter equations

$$
\hat{\boldsymbol{x}}_{\mathrm{S}}[k]=\hat{\boldsymbol{x}}_{\mathrm{S}}[k-1]+\boldsymbol{K}_{\mathrm{S}}[k] \boldsymbol{\Pi}_{\mathrm{S}}^{\mathrm{T}} \boldsymbol{K}[k] \boldsymbol{r}[k] .
$$

In (73) it was first used that following (65) the best a priori estimates for $\boldsymbol{x}_{\mathrm{S}}[k]$ is $\hat{\boldsymbol{x}}_{\mathrm{S}}[k-1]$ and that the measurment residual of the dual Kalman filter can be directly derived from the measurement residual $\boldsymbol{r}[k]$ of the primal Kalman filter. The Kalman gain of the dual Kalman filter follows from the general Kalman filter equations

$$
\boldsymbol{K}_{\mathrm{S}}[k]=\boldsymbol{P}_{\mathrm{S}}[k \mid k-1] \boldsymbol{h}_{\mathrm{S}}[k]\left(\boldsymbol{h}_{\mathrm{S}}^{\mathrm{T}}[k] \boldsymbol{P}_{\mathrm{S}}[k \mid k-1] \boldsymbol{h}_{\mathrm{S}}[k]+\sigma_{\mathrm{S}}^{2}\right)^{-1}
$$

with a priori error covariance matrix estimate

$$
\boldsymbol{P}_{\mathrm{S}}[k \mid k-1]=\boldsymbol{P}_{\mathrm{S}}[k-1]+\boldsymbol{Q}_{\mathrm{S}},
$$

and the error covariance estimate

$$
\boldsymbol{P}_{\mathrm{S}}[k]=\left(\boldsymbol{I}-\boldsymbol{K}_{\mathrm{S}}[k] \boldsymbol{h}_{\mathrm{S}}^{\mathrm{T}}[k]\right) \boldsymbol{P}_{\mathrm{S}}[k \mid k-1] .
$$

\section{AR Noise Variance Estimation}

As the LOS phase propagation is dominated by the satellite movement we assume that $\boldsymbol{Q}_{0}$ is known a priori, i.e. from ephemeris data. However, also the AR process noise covariance matrices $\boldsymbol{Q}_{\gamma}[k], \boldsymbol{Q}_{\phi}[k]$ or more precisely their variance entries $\sigma_{\gamma}^{2}$ and $\sigma_{\phi}^{2}$ must be known. They are estimated using

$$
\begin{aligned}
\boldsymbol{\Pi}_{\mathrm{S}} \boldsymbol{P}[k] \boldsymbol{\Pi}_{\mathrm{S}}^{\mathrm{T}}= & \boldsymbol{\Pi}_{\mathrm{S}}\left(\boldsymbol{A P}[k-1] \boldsymbol{A}^{\mathrm{T}}+\boldsymbol{Q}\right. \\
& -\boldsymbol{K}[k] \boldsymbol{H}[k] \boldsymbol{P}[k \mid k-1]) \boldsymbol{\Pi}_{\mathrm{S}}^{\mathrm{T}} .
\end{aligned}
$$


Solving for $\sigma_{\mathrm{S}}^{2}=\Pi_{\mathrm{S}} \boldsymbol{Q} \Pi_{\mathrm{S}}^{\mathrm{T}}$ yields

$$
\begin{aligned}
\sigma_{\mathrm{S}}^{2}= & \boldsymbol{\Pi}_{\mathrm{S}}\left(\boldsymbol{P}[k]-\boldsymbol{A P}[k-1] \boldsymbol{A}^{\mathrm{T}}\right. \\
& +\boldsymbol{K}[k] \boldsymbol{H}[k] \boldsymbol{P}[k \mid k-1]) \boldsymbol{\Pi}_{\mathrm{S}}^{\mathrm{T}} .
\end{aligned}
$$

The term $\boldsymbol{K}[k] \boldsymbol{H}[k] \boldsymbol{P}[k \mid k-1]$ can be rewritten

$$
\boldsymbol{K}[k] \boldsymbol{H}[k] \boldsymbol{P}[k \mid k-1]=\boldsymbol{K}[k] \boldsymbol{S}[k] \boldsymbol{K}[k]^{\mathrm{T}} .
$$

Therefore $\sigma_{\mathrm{S}}^{2}$ can be iteratively estimated with

$$
\hat{\sigma}_{\mathrm{S}}^{2}=\mu \hat{\sigma}_{\mathrm{S}}^{2}[k-1]+(1-\mu) \boldsymbol{\Pi}_{\mathrm{S}} \boldsymbol{V}[k] \boldsymbol{\Pi}_{\mathrm{S}}^{\mathrm{T}},
$$

where the forgetting factor $\mu \in \mathbb{R}$ is chosen close to 1 and

$$
\boldsymbol{V}[k]=\boldsymbol{P}[k]-\boldsymbol{A P}[k-1] \boldsymbol{A}^{\mathrm{T}}+\boldsymbol{K}[k] \boldsymbol{r}[k] \boldsymbol{r}[k]^{\mathrm{T}} \boldsymbol{K}[k]^{\mathrm{T}}
$$

as $\boldsymbol{S}[k]$ is the covariance matrix of $\boldsymbol{r}[k]$.

\section{E. AR Model Order Estimation}

In general $P_{\gamma}$ and $P_{\phi}$ are unknown and must be estimated, for example with the Akaike information criterion. However, other authors showed, based on simulated data [14], that order $P_{\gamma}=2$ and $P_{\phi}=1$ are appropriate in the case of moderate ionospheric scintillations. Therefore, we use these values.

\section{Verification with Simulated Data}

In the following we verify the functionality of the proposed dual Kalman Filter approach at the example of a scenario with the GPS L1 C/A signal. The correlation time is $T=10 \mathrm{~ms}$. The scintillation phase and amplitude are generated with the CSM. We evaluate the root mean squared error (RMSE) over 100 Monte Carlo Runs over different LOS and scintillation realizations. The results of the proposed algorithm (Dual KF) are compared to three alternative algorithms

Given Parameter KF: Kalman filter which uses the same offline estimates of the AR parameters of $\phi_{\mathrm{S}}[k]$ and $\gamma_{\mathrm{S}}[k]$ during the whole tracking process.

Yule KF: Kalman filter which does not track $\gamma_{\mathrm{S}}[k]$ and uses the Yule-Walker equations to estimate the AR parameters of $\phi_{\mathrm{S}}$. Instead of an extended Kalman filter an atan2 phase discriminator with linear measurement equation is used.

Classic KF: Standard Kalman filter, which does not track $\phi_{\mathbf{S}}[k]$ and $\gamma_{\mathbf{S}}[k]$, and uses an atan2 phase discriminator.

\section{A. Overall Phase Tracking}

Fig. 2 shows the RMSE of $\bmod (\phi[k], 2 \pi)$ for two scenarios with $S_{4}=0.6$ and $S_{4}=0.8$, respectively. The modulo operator is used, such that non detected cycle slips do not increase the RMSE. The other parameters are the same in both scenarios, i.e. the decorrelation time is $\tau_{0}=0.1 \mathrm{~s}$, the carrier-to-noise density is $\mathrm{C} / \mathrm{N}_{0}=45 \mathrm{~dB}$ and the Doppler drift rate variance is $\sigma_{\dot{a}}^{2}=2 \cdot 10^{-1}$. In the first $30 \mathrm{~s}$ the scintillation is turned off and only the LOS phase has to be tracked. After $30 \mathrm{~s}$ scintillation is turned on. In the case of no scintillation the Given Parameter $K F$ algorithm here shows a significantly higher error than Classic KF, Dual KF, and Yule $K F$ as it always assumes scintillations and tries to estimate a scintillation phase. It is therefore not the optimum estimator in the case of no scintillations. When the scintillations are turned on Classic KF has the highest RMSE and the Given

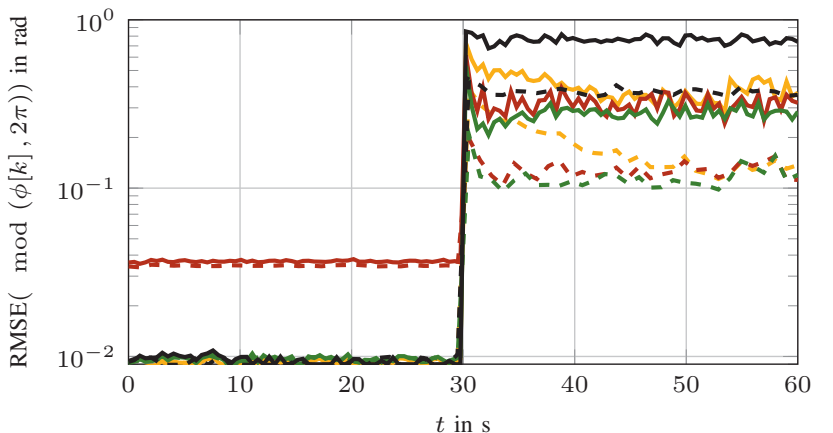

$$
\begin{array}{|ll|}
\hline \text { - }- \text { - Dual KF }\left(S_{4}=0.6\right) & \text { Dual KF }\left(S_{4}=0.8\right) \\
\text { - }- \text { - Given Parameter KF }\left(S_{4}=0.6\right) & \text { Given Parameter KF }\left(S_{4}=0.8\right) \\
\text { - - - Yule KF }\left(S_{4}=0.6\right) & \text { Yule KF }\left(S_{4}=0.8\right) \\
- \text { - - Classic KF }\left(S_{4}=0.6\right) & \text { Classic KF }\left(S_{4}=0.8\right) \\
\hline
\end{array}
$$

Fig. 2. RMSE of phase $\phi[k]=\phi_{0}[k]+\phi_{\mathrm{S}}[k]$ for different $S_{4}$ and algorithms

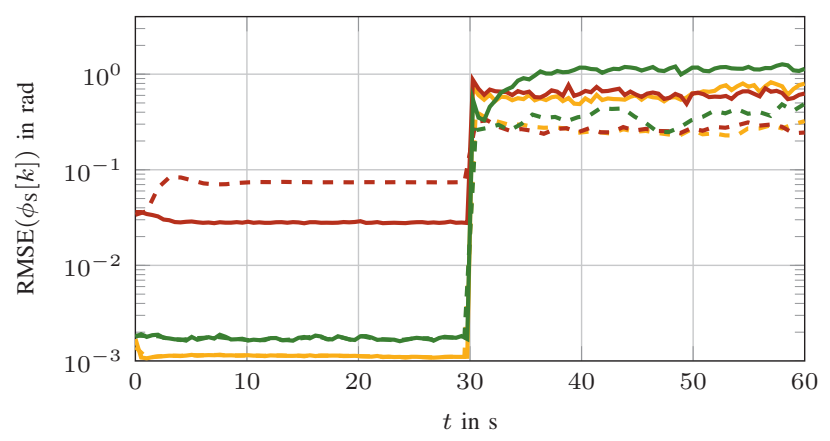

$$
\begin{array}{|ll}
\hline \text { - }=\text { - Dual KF }\left(S_{4}=0.6\right) & \text { Dual KF }\left(S_{4}=0.8\right) \\
\text { - }- \text { - Given Parameter KF }\left(S_{4}=0.6\right) & \text { Given Parameter KF }\left(S_{4}=0.8\right) \\
- \text { - - Yule KF }\left(S_{4}=0.6\right) & \text { Yule KF }\left(S_{4}=0.8\right)
\end{array}
$$

Fig. 3. RMSE of scintillation phase $\phi_{\mathrm{S}}[k]$ for different $S_{4}$ and algorithms.

Parameter $K F$ and the Yule $K F$ algorithms converge fast to a common error, while the proposed algorithm shows a lower convergence rate. However, when we consider the number of Monte Carlo runs with at least one cycle slip, the proposed algorithm shows with $0 \%$ cycle slips in the case of $S_{4}=0.6$ and $25 \%$ for $S_{4}=0.8$ a smaller absolute phase error than the Yule $K F\left(6 \%\right.$ for $S_{4}=0.6$ and $60 \%$ for $S_{4}=0.8$ ) and the Given Parameter $K F$ (5\% for $S_{4}=0.6$ and $40 \%$ for $S_{4}=0.8$ ).

\section{B. Scintillation Phase Tracking}

Fig. 3 shows the RMSE for the estimate of $\Phi_{\mathrm{S}}[k]$. The Classic $K F$ is not shown here as it does not explicitly estimate $\Phi_{S}[k]$. In the case of scintillations, the Given Parameter $K F$ algorithm and the proposed algorithm Dual KF show a lower error than Yule $K F$. This difference also increases with increasing $S_{4}$. For monitoring applications, the proposed algorithm therefore offers the better performance.

\section{Scintillation Amplitude Tracking}

Fig. 4 shows the estimation error for $\gamma_{S}[k]$ for the Given Parameter KF and the proposed algorithm Dual KF. The Dual $K F$ achieves the estimation performance of the algorithm with known AR model parameters, i.e. it is able to estimate the AR model parameters of the scintillation amplitude. 




\begin{tabular}{|ll|}
\hline - $=$ - Dual KF $\left(S_{4}=0.6\right)$ & Dual KF $\left(S_{4}=0.8\right)$ \\
- - - Given Parameter KF $\left(S_{4}=0.6\right)$ & Given Parameter KF $\left(S_{4}=0.8\right)$
\end{tabular}

Fig. 4. RMSE of scintillation amplitude $\gamma_{S}[k]$ for different $S_{4}$ and algorithms.

\section{CONCLUSIONS}

We have presented a dual Kalman filtering based algorithm for GNSS phase tracking. The algorithm uses separate state space models to track the LOS phase and the scintillation phase and amplitude with a linear quadratic Gaussian controller, i.e. a Kalman filter as state estimator and a LQR to design the feedback loop. The LOS phase is modeled by a Wiener process while the scintillation phase and amplitude are modeled by an AR process. For the estimation of the AR parameters of scintillation phase and amplitude a second Kalman filter was introduced. The second Kalman filter takes the scintillation phase and amplitude estimates of the first Kalman filter as measurements to track the AR parameters. Simulation results with the CSM show that the presented algorithm is able to track the overall phase and scintillation phase and amplitude with the same performance as a comparable algorithm which uses offline estimates of the AR parameters. As the AR parameters reflect the temporal correlation of the scintillation phase and amplitude, they can also be used in monitoring applications.

\section{REFERENCES}

[1] S. Ganguly, A. Jovancevic, A. Brown, M. Kirchner, S. Zigic, T. Beach, and K. M. Groves, "Ionospheric scintillation monitoring and mitigation using a software GPS receiver," Radio Science, vol. 39, no. 1, pp. 1-9, 2004.

[2] F. Legrand, C. Macabiau, J.-L. Issler, L. Lestarquit, and C. Mehlen, "Improvement of pseudorange measurements accuracy by using fast adaptive bandwidth lock loops," in ION GPS 2000, Salt Lake City, United States, Sep. 2000, pp. 2346 - 2356.

[3] S. Skone, G. Lachapelle, D. Yao, W. Yu, and R. Watson, "Investigating the Impact of Ionospheric Scintillation using a GPS Software Receiver," in Proceedings of the 18th International Technical Meeting of the Satellite Division of The Institute of Navigation (ION GNSS 2005), Long Beach, CA, Sep. 2005, pp. 1126-1137.

[4] R. Xu, Z. Liu, and W. Chen, "Improved FLL-assisted PLL with in-phase pre-filtering to mitigate amplitude scintillation effects," GPS Solutions, vol. 19, pp. 263-276, 2015.

[5] P. Henkel, G. Kaspar, and C. Günther, "Multifrequency, mulitsatellite vector phase-locked loop for robust carrier tracking," IEEE Journal on Selected Topics in Signal Processing, vol. 3, pp. 674-681, 2009.

[6] F. van Graas, A. Soloviev, M. U. de Haag, and S. Gunawardena, "Closed-Loop Sequential Signal Processing and Open-Loop Batch Processing Approaches for GNSS Receiver Design," IEEE Journal of Selected Topics in Signal Processing, vol. 3, pp. 571-586, 2009.
[7] Z. He and M. G. Petovello, "Performance Comparison of Kalman Filter and Maximum Likelihood Carrier Phase Tracking for Weak GNSS Signals," in International Conference on Indoor Positioning and Indoor Navigation, Banff, Canada, Oct. 2015.

[8] J. T. Curran, M. Bavaro, A. Morrison, and J. Fortuny, "Developing a Multi-Frequency for GNSS-Based Scintillation Monitoring Receiver," in Proceedings of the 27th International Technical Meeting of The Satellite Division of the Institute of Navigation (ION GNSS+ 2014), Tampa, Florida, Sep. 2014, pp. 1142-1152.

[9] M. L. Psiaki, T. E. Humphreys, A. P. Cerruti, S. P. Powell, and P. M. Kintner Jr, "Tracking L1 C/A and L2C Signals through Ionospheric Scintillations," in Proceedings of the 20th International Technical Meeting of the Satellite Division of The Institute of Navigation (ION GNSS 2007), Fort Worth, TX, Sep. 2007, pp. 246-268.

[10] V. Barreau, W. Vigneau, C. Macabiau, and L. Deambrogio, "Kalman Filter based robust GNSS signal tracking algorithm in presence of ionospheric scintillations," in 2012 6th ESA Workshop on Satellite Navigation Technologies (Navitec 2012) European Workshop on GNSS Signals and Signal Processing, Noordwijk, Netherlands, Dec. 2012, pp. $1-8$.

[11] M. L. Psiaki and H. Jung, "Extended Kalman Filter Methods for Tracking Weak GPS Signals," in Proceedings of the 15th International Technical Meeting of the Satellite Division of The Institute of Navigation (ION GPS 2002), Portland, OR, Sep. 2002, pp. 2539-2553.

[12] A. Van Dierendonck, J. Klobuchar, and Q. Hua, "Ionospheric scintillation monitoring using commercial single frequency C/A code receivers," in Proceedings of the 6th International Technical Meeting of the Satellite Division of The Institute of Navigation (ION GPS 1993), vol. 93, Salt Lake City, UT, Sep. 1993, pp. 1333-1342.

[13] J. Vilá-Valls, J. López-Salcedo, and G. Seco-Granados, "An Interactive Multiple Model Approach for Robust GNSS Carrier Phase Tracking under Scintillation Conditions," in Proceedings of the IEEE International Conference on Acoustics, Speech and Signal Processing, ICASSP 2013, Vancouver, BC, Canada, May 2013.

[14] J. Vilá-Valls, C. Fernandez-Prades, J. López-Salcedo, and G. SecoGranados, "Adaptive GNSS Carrier Tracking Under Ionospheric Scintillation: Estimation vs. Mitigation," IEEE Communications Letters, vol. 19, pp. 961-964, May 2015.

[15] S. Locubiche-Serra, G. Seco-Granados, and J. A. López-Salcedo, "Doubly-adaptive autoregressive Kalman filter for GNSS carrier tracking under scintillation conditions," in 2016 International Conference on Localization and GNSS (ICL-GNSS), Barcelona, Spain, Aug. 2016, pp. $1-6$.

[16] D. Labarre, E. Grivel, Y. Berthoumieu, E. Todini, and M. Najim, "Consistent estimation of autoregressive parameters from noisy observations based on two interacting Kalman filters," Signal Processing, vol. 86, no. 10, pp. 2863-2876, 2006.

[17] T. E. Humphreys, M. Psiaki, and P. Kintner, "Modeling the Effects of Ionosperic Scintillation on GPS Carrier Phase Tracking," IEEE Transactions on Aerospace and Electronic Systems, vol. 46, no. 4, pp. 1624-1637, Oct. 2010.

[18] A. Van Dierendonck, "GPS receivers," Global Positioning System: Theory and Applications., vol. 1, pp. 329-407, 1996.

[19] S. M. Kay, Fundamentals of Statistical Signal Processing, Volume 1: Estimation Theory. Prentice-Hall PTR, 1993.

[20] C. Zucca and P. Tavella, "The clock model and its relationship with the allan and related variances," IEEE Transactions on Ultrasonics, Ferroeletrics, and Frequency Control, vol. 52, no. 2, pp. 289-296, Feb. 2005.

[21] E. J. Fremouw, R. C. Livingston, and D. A. Miller, "On the statistics of scintillating signals," Journal of Atmospheric and Terrestrial Physics, vol. 42, pp. 717-713, 1980.

[22] H. E. Whitney, J. A. Aarons, R. S. Allen, and D. R. Seemann, "Estimation of the cumulative amplitude probability distribution function of ionospheric scintillation," Radio Science, vol. 12, pp. 1095-1104, 1972.

[23] M. Athans, "The role and use of the stochastic linear-quadratic-gaussian problem in control system design," IEEE Transactions on Automatic Control, vol. 16, no. 6, pp. 529-552, 1971.

[24] R. Curry, "Separation theorem for nonlinear measurements," IEEE Transactions on Automatic Control, vol. 14, no. 5, pp. 561-564, 1969. 